\title{
Interferon- $\gamma$ inhibits group B Streptococcus survival within human endothelial cells
}

\author{
Viviane de Oliveira Freitas Lione', Michelle Hanthequeste Bittencourt dos Santos², \\ Jessica Silva Santos de Oliveira², Ana Luiza Mattos-Guaraldi ${ }^{3}$, Prescilla Emy Nagao $^{2}{ }^{+}$
'Laboratório de Bioensaios Farmacêuticos, Faculdade de Farmácia, Universidade Federal do Rio de Janeiro, Rio de Janeiro, RJ, Brasil
${ }^{2}$ Departamento de Biologia Celular, Instituto de Biologia Roberto Alcântara Gomes ${ }^{3}$ Disciplina de Microbiologia e Imunologia, Faculdade de Ciências Médicas, Universidade do Estado do Rio de Janeiro, Rio de Janeiro, RJ, Brasil

Endothelial dysfunction is a major component of the pathophysiology of septicaemic group B Streptococcus (GBS) infections. Although cytokines have been shown to activate human umbilical vein endothelial cells (HUVECs), the capacity of interferon (IFN)- $\gamma$ to enhance the microbicidal activity of HUVECs against GBS has not been studied. We report that the viability of intracellular bacteria was reduced in HUVECs activated by IFN- $\gamma$. Enhanced fusion of lysosomes with bacteria-containing vacuoles was observed by acid phosphatase and the colocalisation of Rab-5, Rab-7 and lysosomal-associated membrane protein-1 with GBS in IFN- $\gamma$-activated HUVECs. IFN- $\gamma$ resulted in an enhancement of the phagosome maturation process in HUVECs, improving the capacity to control the intracellular survival of GBS.

Key words: group B Streptococcus - IFN- $\gamma$ - survival

Group B Streptococcus (GBS) bacteria are a leading cause of life-threatening infections in neonates and young infants (Baker 2013). Invasive neonatal GBS infection has either an early (usually within the first $24 \mathrm{~h}$ after birth) or a late (7 days after birth) onset and common manifestations of GBS disease in neonates include pneumonia, septicaemia, meningitis, bacteraemia and bone or joint infections (Baker 2013). Invasive disease caused by GBS has also been recognised in adults (Schwartz et al. 1991, Gardam et al. 1998).

Endothelial dysfunction is a major component of the pathophysiology of septicaemic GBS infection (Lembo et al. 2010, Beyrich et al. 2011). Therefore, analysis of the interaction between GBS and endothelial cells is of major interest, especially during septic and haemorrhagic complications in newborns and adults.

Endothelial cells have been viewed as comprising the passive structural lining of blood vessels; however, it is now evident that upon activation by cytokines such as interferon (IFN)- $\gamma$, these cells can impact inflammatory reactions and immune responses (Mantovani et al. 1992). Although endothelial cells are not true phagocytes, they share a number of functional similarities with macrophages upon stimulation, such as exhibiting Fc receptormediated phagocytosis, expressing class II major histocompatibility complex antigens and presenting antigens to lymphocytes (Rothermel et al. 2004, Beyrich et al. 2011).

doi: 10.1590/0074-0276140201

Financial support: FAPERJ, CNPq, UERJ

+Corresponding author: pnagao@pq.cnpq.br

Received 5 June 2014

Accepted 30 September 2014
IFN- $\gamma$ is a proinflammatory cytokine that enhances host defences against a variety of pathogens. The exogenous administration of IFN- $\gamma$ protects against septicaemia in a mouse model of Staphylococcus aureus infection, leading to more efficient bactericidal activity (Zhao et al. 1998). Previous studies have shown that IFN- $\gamma$ exerts beneficial effects on GBS-induced arthritis by activating phagocytic cells and decreasing bacterial load (Puliti et al. 2002). Nevertheless, the capacity of IFN- $\gamma$ to enhance the microbicidal activity of endothelial cells against GBS has not been studied.

Pathogen engulfment by phagocytic vacuoles is an essential component of the innate immune response (Underhill \& Ozinsky 2002). Following formation, vacuoles or nascent phagosomes acquire microbicidal properties through a maturation process that involves sequential fusion events with compartments of the endocytic pathway, culminating in the formation of a hybrid organelle: the phagolysosome (Vieira et al. 2001, Underhill \& Ozinsky 2002). Similarly, it is thought that Rab GTPases orchestrate the sequence of fusion events in the endocytic pathway (Vieira et al. 2003). Accordingly, Rab-5 has been detected in early phagosomes, where it transiently resides, directing fusion events and Rab-7 has been detected at later maturation stages (Vieira et al. 2001, 2003, Underhill \& Ozinsky 2002). Rab-7 mediates the fusion of lysosomes to phagosomes and Rab-5 acts upstream of Rab-7 by tethering early endosomes and nascent phagosomes (Guo \& Wang 2010). Lysosomalassociated membrane protein-1 (LAMP-1) is among the most abundant lysosomal membrane proteins and is expressed mainly in the endosome-lysosomal membrane (Saftig \& Klumperman 2009).

In the present study, we investigated whether IFN- $\gamma$ contributes to bacteria killing via human umbilical vein endothelial cells (HUVECs). We show that these cells 
can be activated by IFN- $\gamma$ to kill GBS through increased vacuolar recruitment of Rab-5, Rab-7 and Lamp-1 during the intermediate maturation stages of GBS-containing vacuoles (GBS-CVs). This process has not yet been described for GBS.

GBS strain 90356 (serotype III, a central nervous system isolate), a strain identified as GBS and serotyped as described previously (Lancefield 1934), was used in this study.

Primary HUVECs were obtained by treating umbilical veins with $0.1 \%$ collagenase IV solution (Sigma Chemical Co, USA), as previously described (Jaffe et al. 1973). Confluent HUVECs were infected with GBS [5 x $10^{7}$ colony-forming unit (CFU)] at 1:100 multiplicity of infection for $0 \mathrm{~h}, 0.5 \mathrm{~h}, 1 \mathrm{~h}$ and $2 \mathrm{~h}$ and then incubated with $5 \% \mathrm{CO}_{2}$ at $37^{\circ} \mathrm{C}$. The total GBS association (intracellular plus surface adherence) was estimated by plating epithelial lysates on $\mathrm{BAB} /$ blood plates containing $5 \%$ sheep defibrinated blood and counting the resulting colonies. After each period of incubation, the infected monolayers were incubated for an additional period of $2 \mathrm{~h}$ in M199 containing bactericidal amounts of gentamicin $(100 \mu \mathrm{g} /$ $\mathrm{mL})$ and penicillin $\mathrm{G}(5 \mu \mathrm{g} / \mathrm{mL})$. The number of internalised bacteria was determined as outlined above. The adherence rates were determined as follows: [CFU of total cell-associated (intracellular viable plus surface adherent) GBS-CFU intracellular GBS] (Santos et al. 2003).

Acid phosphatase activity was detected according to the method described by Teixeira et al. (2001). Identification of the lysosomal-endocytic pathway compartments involved in the internalisation of GBS was performed by double labelling with anti-Rab-5, anti-Rab-7 or antiLAMP-1 antibodies and 4,6-diamidino-2-phenylindole dihydrochloride (DAPI) (Molecular Probes, USA) at 0.5 $\mathrm{h}, 1 \mathrm{~h}$ and $2 \mathrm{~h}$ post-infection (p.i.) with GBS. The infected HUVECs were first fixed with 3.5\% formaldehyde and incubated with peptidoglycan (PGN) [phosphate-buffered saline (PBS), $0.15 \%$ gelatine, $0.1 \%$ sodium azide] plus $0.1 \%$ of saponin for $30 \mathrm{~min}$. The cells were then stained with the monoclonal antibody at 1:50 diluted in $\mathrm{PGN} / 0.1 \%$ saponin for $1 \mathrm{~h}$ at room temperature, washed three times with PBS and then incubated with fluorescein-labelled goat anti-rabbit IgG (Sigma), diluted 1:100 in PGN for $1 \mathrm{~h}$ in the presence of $10 \mathrm{mM}$ DAPI. Confocal images were obtained using the Bio-Rad 1024 UV system coupled to a Zeiss Axiovert 100 microscope or a Leica TCS SP5 II system. All experiments were performed in duplicate and repeated at least three times. A statistical analysis was performed using Sigma Stat v.1.0 (Jandel Scientific) and Student's $t$ test. A p-value $\leq 0.05$ was considered significant.

Endothelial cells have been classically viewed as passive members of the immune system, acting as barriers to the influx of microbes and secreting factors that attract true phagocytes to the sites of bacterial invasion. We have shown in a previous study that human endothelial cells internalise GBS, but do not efficiently eliminate internalised bacteria (Lione et al. 2010), which most likely and partially stems from the low indices of endosomelysosome fusion observed in GBS-infected HUVECs.
The adherence and intracellular viability of GBS in endothelial cells were assessed by penicillin/gentamicin exclusion. Fig. 1A shows that the control cells presented higher counts of adherent bacteria than the cytokinestimulated HUVECs $(p<0.05)$. Similarly, the stimulated cells showed significantly less viable bacteria than the control cells $(\mathrm{p}<0.01)$ (Fig. 1B). Our results show that GBS bacteria attach to the surface of HUVECs and are ingested through phagocytosis, forming a membranebound vacuole. This process could explain the decreased adherence and viability shown in Fig. 1A, B. Some of the ingested bacteria displayed significant morphological changes, clearly indicating that they were killed by the HUVECs (data not shown). The observation of labelled bacteria-containing vacuoles indicates fusion between phagosomes and lysosomes.

A classic enzyme marker for lysosomes was detected inside the phagocytic vacuoles. The reaction product, which suggests enzyme activity, was observed at higher levels in the HUVECs treated with IFN- $\gamma$ than in the control cells (Fig. 1C). Through the presence of acid phosphatase, a classic enzyme marker of lysosomes, our ultrastructural observations show lysosomal fusion with bacteria-containing vacuoles. We demonstrate that IFN- $\gamma$ can modulate the bactericidal activity of HUVECs; however, the process that initiates the killing of the bacteria remains unclear. These results are consistent with those of other investigators who observed a significant inhibition of bacterial growth and a concomitant production of IFN- $\gamma$ in GBS-infected neonatal mouse cells treated with interleukin-12 (Puliti et al. 2002).

We observed that GBS-CVs were enriched in Rab-5, Rab-7 and LAMP-1 at $0.5 \mathrm{~h}, 1 \mathrm{~h}$ and $2 \mathrm{~h}$ p.i. in IFN- $\gamma$ treated HUVECs. As no statistically significant difference was observed between the results at $1 \mathrm{~h}$ and $2 \mathrm{~h}$ p.i., only results from $0.5 \mathrm{~h}$ and $2 \mathrm{~h}$ are presented in Fig. 2 .

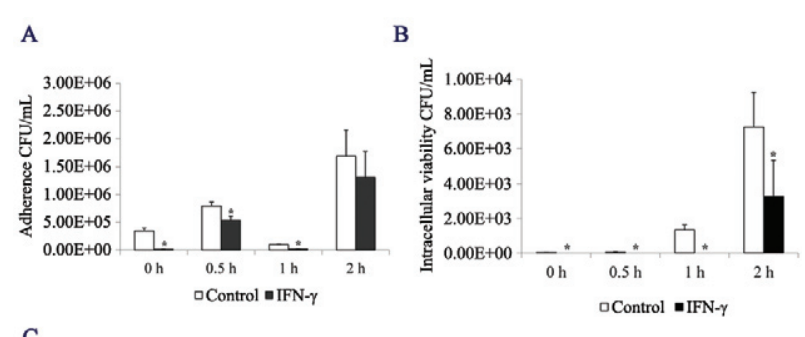

$\mathrm{C}$

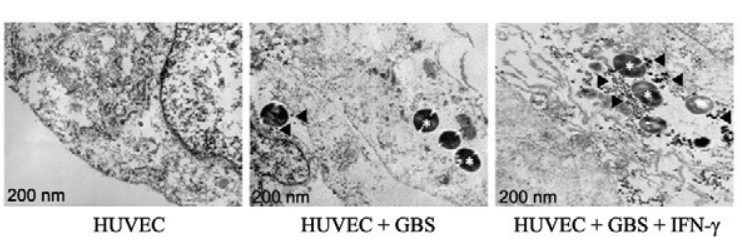

Fig. 1: adherence (A) and intracellular viability (B) of group B Streptococcus (GBS) 90356-III in human umbilical vein endothelial cell (HUVEC) cells. Results are presented as means \pm standard deviations from at least three independent experiments in duplicate wells. Data are presented in a graphic log scale. Micrographs of GBS (asterisks) inside the HUVEC (C). The pictures show fusion of lysosomes with phagosomes through the detection of acid phosphatase activity (arrows). CFU: colony-forming unit; IFN: interferon. 
A

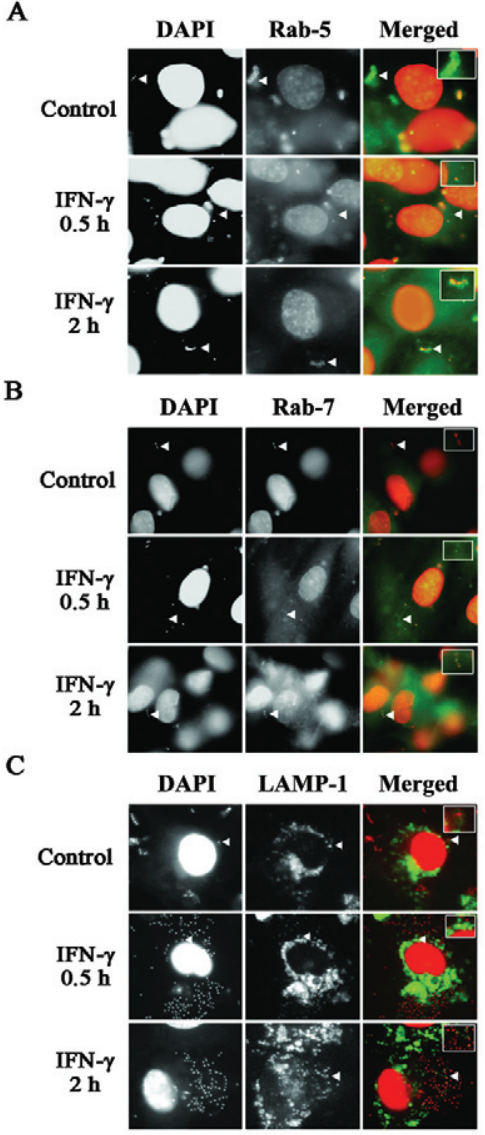

Fig. 2: infection of human umbilical vein endothelial cell (HUVEC) by the group B Streptococcus (GBS) 90356-III strain (A-C) after 0.5 $\mathrm{h}$ and $2 \mathrm{~h}$ post-infection in HUVEC untreated (control) or interferon (IFN)- $\gamma$-treated cells. Cells were anti Rab-5, Rab-7 or lysosomal-associated membrane protein-1 (LAMP-1) antibody labelled and 4,6diamidino-2-phenylindole dihydrochloride (DAPI) stained followed by fluorescein isothiocyanate-labelling with secondary antibody. Arrows point to GBS colocalised with endosomal/lysosomal markers.

Laser-scanning confocal microscopy revealed that GBS colocalises with Rab-5 and Rab-7 at $0.5 \mathrm{~h}$ and $2 \mathrm{~h}$ p.i. (Fig. 2A, B); colocalisation of LAMP-1 was also observed with an increased number of bacteria within vacuoles at $2 \mathrm{~h}$ p.i. (Fig. 2C). Similar results were observed in the control cells (after $0.5 \mathrm{~h}, 1 \mathrm{~h}$ and $2 \mathrm{~h}$ ).

In addition, our data show the transient accumulation of the late endosomal/lysosomal markers Rab-5 and Rab-7 in maturing GBS-CVs during HUVEC GBS infection. Moreover, we also show GBS-CV recruitment of LAMP-1, demonstrating that GBS-CVs move along the endocytic pathway and fuses with lysosomes. The data demonstrating the interaction between intermediate GBS-CVs and the late endosomal/lysosomal compartment suggest that GBS is being killed by the IFN- $\gamma$-treated HUVECs. Indeed, this study shows, for the first time, the colocalisation of GBS and endosomal markers in endothelial cells treated with IFN- $\gamma$. Similarly, the treatment of macrophages with IFN- $\gamma$ induces the biosynthesis of Rab-5 and, in parallel, accelerates the intracellular killing of pathogens such as Listeria monocytogenes (Alvarez-Dominguez \& Stahl 1998); Rab-5, which is recruited rapidly and transiently to the phagosome, was also found to be essential for the recruitment of Rab-7 and the progression to phagolysosomes for this microorganism (Alvarez-Dominguez \& Stahl 1998).

Thus, we conclude that the expression of Rab-5, Rab-7 and Lamp-1 in HUVECs resulted in their ability to enhance the phagosome maturation process and increase bacterial killing. Future studies analysing genes that are regulated by IFN- $\gamma$ may provide important knowledge about the proteins required for GBS to increase the phagosome maturation process.

Our results show that IFN- $\gamma$ has a bactericidal effect through increased maturation of the endocytic pathway. These results indicate that IFN- $\gamma$ should be investigated as a way to better control GBS infections.

\section{REFERENCES}

Alvarez-Dominguez C, Stahl PD 1998. Interferon-gamma selectively induces Rab5a synthesis and processing in mononuclear cells. $J$ Biol Chem 273: 33901-33904.

Baker CJ 2013. The spectrum of perinatal group B streptococcal disease. Vaccine 31 (Suppl. 4): D3-D6.

Beyrich C, Löffler J, Kobsar A, Speer CP, Kneitz S, Eigenthaler M 2011. Infection of human coronary artery endothelial cells by group B Streptococcus contributes to dysregulation of apoptosis, hemostasis and innate immune responses. Mediators Inflamm 2001: 971502.

Gardam MA, Low DE, Saginur R, Miller MA 1998. Group B streptococcal necrotizing fasciitis and streptococcal toxic shock-like syndrome in adults. Arch Intern Med 158: 1704-1708.

Guo P, Wang X 2010. Rab GTPases act in sequential steps to regulate phagolysosome formation. Small GTPases 1: 170-173.

Jaffe EA, Nachamann RL, Becker CG, Minick CR 1973. Culture of human endothelial cells derived from umbilical cord veins. Identification by morphologic and immunologic criteria. J Clin Invest 52: $2745-2756$.

Lancefield RC 1934. A serological differentiation of specific types of bovine hemolytic streptococci (Group B). J Exp Med 59: 441-458.

Lembo A, Gurney MA, Burnside K, Banerjee A, de los Reyes M, Connelly JE, Lin WJ, Jewell KA, Vo A, Renken CW, Doran KS, Rajagopal L 2010. Regulation of CovR expression in Group B Streptococcus impacts blood-brain barrier penetration. Mol Microbiol 77: 431-443.

Lione VOF, Santos MHB, Carvalho TMU, Hirata Jr R, Mattos-Guaraldi AL, Mortara RA, Nagao PE 2010. Fever temperature enhances mechanisms of survival of Streptococcus agalactiae within human endothelial cells. Int J Mol Med 26: 511-516.

Mantovani A, Bussolino F, Dejana E 1992. Cytokine regulation of endothelial cell functions. FASEB J 6: 2591-2599.

Puliti M, von Hunolstein C, Bistoni F, Mosci P, Orefici G, Tissi L 2002. The beneficial effect of interleukin-12 on arthritis induced by Group B streptococci is mediated by interferon- $\gamma$ and interleukin-10 production. Arthritis Rheum 46: 806-817.

Rothermel AL, Wang Y, Schechner J, Mook-Kanamori B, Aird WC, Pober JS, Tellides G, Johnson DR 2004. Endothelial cells present antigens in vivo. BMC Immunol 16: 1-15. 
Saftig P, Klumperman J 2009. Lysosome biogenesis and lysosomal membrane proteins: trafficking meets function. Nat Rev Mol Cell Biol 10: 623-635.

Santos GS, Miyazaki NH, Mattos-Guaraldi AL, Nagao PE 2003. The effects of interferon-gamma and transforming growth factor-beta on adherence and survival of group B Streptococcus type III strains in ECV304 cells. Int J Mol Med 11: 401-406.

Schwartz B, Schuchat A, Oxtoby MJ, Cochi SL, Hightower A, Broome CV 1991. Invasive group B streptococcal disease in adults. A population-based study in metropolitan Atlanta. JAMA 266: 1112-1114.

Teixeira CF, Azevedo NL, Carvalho TM, Fuentes J, Nagao PE 2001. Cytochemical study of Streptococcus agalactiae and macrophage interaction. Microsc Res Tech 54: 254-259.
Underhill DM, Ozinsky A 2002. Phagocytosis of microbes: complexity in action. Аnпu Rev Immunol 20: 825-852.

Vieira OV, Botelho RJ, Rameh L, Brachmann SM, Matsuo T, Davidson HW, Schreiber A, Backer JM, Cantley LC, Grinstein S 2001. Distinct roles of class I and class III phosphatidylinositol 3-kinases in phagosome formation and maturation. J Cell Biol 155: 19-25.

Vieira OV, Bucci C, Harrison RE, Trimble WS, Lanzetti L, Gruenberg J, Schreiber AD, Stahl PD, Grinstein S 2003. Modulation of Rab5 and Rab7 recruitment to phagosomes by phosphatidylinositol 3-kinase. Mol Cell Biol 23: 2501-2514.

Zhao YX, Nilsson IM, Tarkowski A 1998. The dual role of interferon- $\gamma$ in experimental Staphylococcus aureus septicaemia versus arthritis. Immunology 93: 80-85. 